\title{
A Feasible Region Contraction Algorithm (Frca) for Solving Linear Programming Problems
}

\author{
E. O. Effanga \\ Department of Mathematics/Statistics and Comp. Science \\ University of Calabar P.M.B. 1115, Calabar, Cross River State, Nigeria \\ E-mail: effanga2005@yahoo.com
}

I. O. Isaac

Department of Mathsematics/Statistics and Comp. Science, University of Calabar

P.M.B. 1115, Calabar, Cross River State, Nigeria

E-mail: idonggrace@yahoo.com

Received: March 10, 2011 Accepted: April 8, 2011 doi:10.5539/jmr.v3n3p159

\begin{abstract}
This paper presents an alternative technique for solving linear programming problems. It is centered on finding a feasible interior point each time the feasible region is contracted, thus generating a sequence of feasible interior points that converge at the optimal solution of linear programming problem. The convergence of the algorithm is proved, and it is shown that the algorithm performs faster in a smaller feasible region.
\end{abstract}

Keywords: Linear programming, Feasible region, Contraction, Interior point

\section{Introduction}

Linear programming is without doubt the most popular tool used in operations research study. A number of solution techniques are available and these include the simplex method developed by Dantzig et al (1956) and the interior point methods initiated by Karmarkar (1984).

The simplex methods and its variants search for the candidate optimal solution in an intelligent fashion by moving from one corner point of the feasible region to another. Although, the simplex methods and its variants have enjoyed widespread acceptance and usage in solving linear programming problems, they are by no means the only good technique. The time and number of iterations required by the simplex methods to reach an optimal solution may be exponential even in small scale linear programming problems thus making the method less efficient (Eiselt et al, 1987).

An attempt to solve linear programming problems in polynomial time facilitated the advent of the interior point methods. Since the dramatic development by Karmarkar (1984), there has been considerable interest in research in the area of interior point methods (Gondzio, 1995; Marsten et al, 1990; Lustig et al, 1994; Todd and Ye, 1990; Todd and Ye, 1998).

Conventionally, the interior point methods start from the initial feasible interior point and follow a central path through the feasible region until an optimum solution is found. This is achieved by converting the given linear programming problem to linear complementarity problem and solved by Newton's method. Although the interior point methods are considered to be highly efficient in practice they have some drawbacks, namely, extensive calculations, many iterations, and large storage space in computer is required. It is therefore necessary to develop a new technique that will overcome these drawbacks.

In other to find a feasible interior point to the system of linear inequalities, Effanga (2010) modified the existing simplex splitting method developed by Levin and Yamnitsky (1982). The modified simplex splitting algorithm is embedded in the feasible region contraction algorithm presented in this paper.

\section{The Idea Behind the Development of FRCA}

Consider the following primal and dual linear programming problems:

Primal:

maximize $Z=c^{T} x$

subject to:

$$
\begin{aligned}
& A x \leq b \\
& x \geq 0
\end{aligned}
$$


Dual:

minimize $Y=b^{T} y$

subject to:

$$
\begin{aligned}
& A^{T} y \geq c \\
& y \geq 0
\end{aligned}
$$

\section{The week duality property:}

If $x^{0}$ is a feasible solution of the primal linear programming problem with the objective function value $Z^{0}$ and $y^{0}$ is a feasible solution of the corresponding dual problem with the objective function value $Y^{0}$, then $Z^{0} \leq Y^{0}$.

\section{The strong duality property:}

If $x^{*}$ is an optimal solution of the primal linear programming problem with the objective function value $Z^{*}$ and $y^{*}$ is an optimal solution of the corresponding dual problem with the objective function value $Y^{*}$, then $Z^{*}=Y^{*}$.

In view of the weak and strong duality properties, it follows that $Z^{0} \leq Z^{*} \leq Y^{0}$.

\section{The Outline of the Feasible Region Contraction Algorithm (FRCA)}

Step 0 Find the feasible interior point $x^{0}$ to the primal problem and the feasible interior point $y^{0}$ to the dual problem using the modified simplex splitting algorithm.

$$
\text { Set } k=0
$$

Step 1 Determine the lower bound $Z^{k}$ and upper bound $Y^{k}$ to the optimum objective function value $Z^{*}$, where

$$
Z^{k}=c^{T} x^{k} \text { and } Y^{k}=b^{T} y^{k}
$$

Step 2 For a small positive number $\varepsilon$,

$$
\text { Is }\left(Y^{k}-Z^{k}\right)<\varepsilon \text { ? }
$$

Yes: Stop, an $\varepsilon$ - optimal solution is found. Set

$$
x^{*}=x^{k} \text { and } Z^{*}=\frac{1}{2}\left(Z^{k}+Y^{k}\right)
$$

No: Go to Step 3.

Step 3 Contract the feasible region by introducing the new constraint

$$
c^{T} x \geq \frac{1}{2}\left(Z^{k}+Y^{k}\right)
$$

Set $k=k+1$

Step 4 Is there a feasible point $x^{f}$ to the updated problem in Step 3?

$$
\begin{aligned}
& \text { Yes: Set } x^{k}=x^{f} \text { and } Z^{k+1}=\frac{1}{2}\left(Z^{k}+Y^{k}\right) \\
& \text { No: Set } x^{k}=x^{k-1} \text { and } Y^{k+1}=\frac{1}{2}\left(Z^{k}+Y^{k}\right)
\end{aligned}
$$

Return to Step 2.

\section{The Convergence of FRCA}

Let $I^{0}:=\left[Z^{0}, Y^{0}\right]$ be the duality gap as defined in the FRCA. Assume that $I^{0}, I^{1}, \cdots, I^{k}$ have been generated. Then denoting $I^{j}:=\left[Z^{j}, Y^{j}\right], j=0,1,2, \cdots, k$, we generate $I^{k+1}$ as follows:

If there is a feasible point with the objective function value greater than $Z^{k+1}$, then $I^{k+1}:=\left[Z^{k+1}, Y^{k}\right]$. But if there is a feasible point with the objective function value less than $Z^{k+1}$, then $I^{k+1}:=\left[Z^{k}, Z^{k+1}\right]$. 


\section{Lemma}

(i) The sequence $\left\{I^{k}\right\}_{k=0}^{\infty}$ is a decreasing sequence of duality gaps. i.e., $I^{k} \supset I^{k+1}, 0 \leq k<\infty$

(ii) The length of $I^{k}$ given by $\frac{Y^{0}-Z^{0}}{2^{k}}$

(iii) $\bigcap_{k=0}^{\infty} I^{k}=\left\{Z^{*}\right\}$, where $Z^{*}$ is the optimal objective function value of the primal linear programming problem.

Proof. Points (i) and (ii) are obvious by our construction.

(iii) For $k=0 Z^{*} \in I^{0}$, by the weak duality property.

$\left\{Z^{k}\right\}_{k=0}^{\infty}$ is a monotonically increasing sequence of primal objective function values, and it is bounded above by $Y^{0}$. It therefore has a finite limit, $L_{1}$.

Also, $\left\{Y^{k}\right\}_{k=0}^{\infty}$ is a monotonically decreasing sequence of dual objective function values, and it is bounded below by $Z^{0}$. It therefore has a finite limit, $L_{2}$.

Now, $L_{2}-L_{1}=\lim _{k \rightarrow \infty}\left(Y^{k}-Z^{k}\right)=\lim _{k \rightarrow \infty}\left(\frac{Y^{0}-Z^{0}}{2^{k}}\right)=0$.

Therefore, $L_{2}=L_{1}=Z^{*}$, by strong duality property.

Theorem 4.1 Let $\left\{X_{i}\right\}_{i=0}^{\infty}$ be a sequence of interior feasible points generated by FRCA, then there exists a subsequence $\left\{X_{i j}\right\}_{j=0}^{\infty}$ that converges at the optimal point $x^{*}$ with the objective function value $Z^{*}$.

Proof. Since the feasible region is compact, a convergence subsequence $\left\{X_{i j}\right\}_{j=0}^{\infty}$ can be selected from the sequence $\left\{X_{i}\right\}_{i=0}^{\infty}$ generated. By the continuity of the expression of the value of a feasible point, $X_{i j} \rightarrow X^{*}$ and $\operatorname{val}\left(X_{i j}\right) \in I_{i} \rightarrow \operatorname{val}\left(X^{*}\right)=Z^{*}$.

Theorem 4.2 The FRCA will terminate after at least $k=\log _{2}\left(\frac{Y^{0}-Z^{0}}{\varepsilon}\right)$ number of iterations.

Proof. Using the stopping rule of FRCA,

it follows that

$$
\text { '( } \left.Y^{k}-Z^{k}\right)<\varepsilon ? ',
$$

That is,

$$
\frac{Y^{0}-Z^{0}}{2^{k}}<\varepsilon .
$$

Therefore,

$$
2^{k} \geq \frac{Y^{0}-Z^{0}}{\varepsilon} .
$$

$$
k \geq \log _{2}\left(\frac{Y^{0}-Z^{0}}{\varepsilon}\right) .
$$

\section{The Effect of Scaling down Right hand side values of constraints on the performance of FRCA}

The Modified Simplex Splitting Algorithm is a nonlinear algorithm which performs faster in a smaller feasible region. This algorithm is performed in steps 0 and step 4 of the FRCA. If the feasible region is therefore reduced in size the decision about the existence of the feasible solution is faster. Hence independent of the main iteration loop, each loop will be faster by itself.

Since the choice of the simplex depends on the right-hand side values, $b_{i}$ 's, of the constraints, it becomes necessary to scale down $b_{i}$ 's by multiplying by a factor $10^{-n}$, for any positive integer $\mathrm{n}$, prior to applying the algorithm. Scaling down the $b_{i}$ 's is equivalent to reduction in size of the feasible region. The optimal solution of the original linear programming problem can be recovered by multiplying the optimal solution of the refined linear programming problem by the factor $10^{n}$.

\section{Example}

Consider the following linear programming problem

minimize $Z=2 x_{1}+3 x_{2}$ 
subject to:

$$
\begin{aligned}
& x_{1}+x_{2} \leq 8 \\
& x_{1}+2 x_{2} \leq 4 \\
& x_{1} \geq 0, x_{2} \geq 0 .
\end{aligned}
$$

Below is the detailed FRCA report of the above linear programming problem.

\section{F. R. C. Algorithm Solver Report}

Solution of LP Problem using the Feasible Region Contraction Algorithm

\section{Primal Problem}

Maximize $Z=2 x_{1}+3 x_{2}$ subject to:
1)
$x_{1}+x_{2} \leq 8$
2)
$x_{1}+2 x_{2} \leq 4$
$x(i) \geq 0, i=1,2$.

\section{Dual Problem}

Minimize $z=8 y_{1}+4 y_{2}$

subject to:

1)

$$
\begin{array}{r}
y_{1}+2 y_{2} \geq 2 \\
1_{1}+y x_{2} \geq 3 \\
y(i) \geq 0, i=1,2 .
\end{array}
$$

\section{Vertices}

Vertex $1=[16,0]$

Vertex $2=[0,16]$

Vertex $3=[0,0]$

\section{Epsilon}

$\varepsilon=0.01$

\section{Results}

$x(0)=[1.8395,0.937]$

$y(0)=[2,2]$

\section{Iteration 1}

Upper $Z(U Z)=24$, Lower $Z(L Z)=6.4901$

$$
(U Z-L Z)=17.5099, \text { is not }<\varepsilon
$$

New Constraint

3)

$$
2 x_{1}+3 x_{2} \geq 15.245
$$

No feasible solution

\section{Iteration 2}

Upper $Z(U Z)=15.245$, Lower $Z(L Z)=6.4901$

$$
(U Z-L Z)=8.755, \text { is not }<\varepsilon
$$

New Constraint

3)

$$
2 x_{1}+3 x_{2} \geq 10.8676
$$

No feasible solution

\section{Iteration 3}

Upper $Z(U Z)=10.8676$, Lower $Z(L Z)=6.4901$ 


$$
(U Z-L Z)=4.3775, \text { is not }<\varepsilon
$$

New Constraint

$$
\text { 3) } \quad 2 x_{1}+3 x_{2} \geq 8.6788
$$

No feasible solution

\section{Iteration 4}

Upper $Z(U Z)=8.6788$, Lower $Z(L Z)=6.4901$

$$
(U Z-L Z)=2.1887, \text { is not }<\varepsilon
$$

New Constraint

3)

$$
2 x_{1}+3 x_{2} \geq 7.5845
$$

Feasible Solution: $x(1)=[3.3146,0.3227]$

\section{Iteration 5}

Upper $Z(U Z)=8.6788$, Lower $Z(L Z)=7.5845$

$$
(U Z-L Z)=1.0944, \text { is not }<\varepsilon
$$

New Constraint

$$
\text { 3) } 2 x_{1}+3 x_{2} \geq 8.1317
$$

No feasible solution

\section{Iteration 6}

Upper $Z(U Z)=8.1317$, Lower $Z(L Z)=7.5845$

$$
(U Z-L Z)=0.5472, \text { is not }<\varepsilon
$$

New Constraint

3)

$$
2 x_{1}+3 x_{2} \geq 7.8581
$$

Feasible Solution: $x(2)=[3.7409,0.1292]$

\section{Iteration 7}

Upper $Z(U Z)=8.1317$, Lower $Z(L Z)=7.8581$

$$
(U Z-L Z)=0.2736, \text { is not }<\varepsilon
$$

New Constraint

3)

$$
2 x_{1}+3 x_{2} \geq 7.9949
$$

Feasible solution: $x(3)=[3.9903,0.0048]$

\section{Iteration 8}

Upper $Z(U Z)=8.1317$, Lower $Z(L Z)=7.9949$

$$
(U Z-L Z)=1.1368, \text { is not }<\varepsilon
$$

New Constraint

3)

$$
2 x_{1}+3 x_{2} \geq 8.0633
$$

No feasible solution

\section{Iteration 9}

Upper $Z(U Z)=8.0633$, Lower $Z(L Z)=7.9949$

$$
(U Z-L Z)=0.0684, \text { is not }<\varepsilon
$$

New Constraint

$$
\text { 3) } 2 x_{1}+3 x_{2} \geq 8.0291
$$

No feasible solution

\section{Iteration 10}

Upper $Z(U Z)=8.0291$, Lower $Z(L Z)=7.9949$ 


$$
(U Z-L Z)=0.0342, \text { is not }<\varepsilon
$$

New Constraint

3)

$$
2 x_{1}+3 x_{2} \geq 8.012
$$

No feasible solution

\section{Iteration 11}

Upper $Z(U Z)=8.012$, Lower $Z(L Z)=7.9949$

$$
(U Z-L Z)=0.0171, \text { is not }<\varepsilon
$$

New Constraint

3)

$$
2 x_{1}+3 x_{2} \geq 8.0034
$$

No feasible solution

\section{Iteration 12}

Upper $Z(U Z)=8.0034$, Lower $Z(L Z)=7.9949$

$$
(U Z-L Z)=0.0085, \text { is not }<\varepsilon
$$

Optimal Solution Found

$Z^{*}=7.9991$

$x^{*}=x(3)=[3.9903,0.0048]$

Now let the right - hand side values of each of the constraints be multiplied by $10^{-1}$. The detailed report of the new problem is given below.

\section{F. R. C. Algorithm Solver Report}

Solution of LP Problem using the Feasible Region Contraction Algorithm

\section{Primal Problem}

Maximize $Z=2 x_{1}+3 x_{2}$

subject to:

$$
\begin{array}{r}
x_{1}+x_{2} \leq 0.8 \\
x_{1}+2 x_{2} \leq 0.4 \\
\text { 2) } \quad \text { x(i) } \geq 0, i=1,2 .
\end{array}
$$

\section{Dual Problem}

Minimize $Z=0.8 y_{1}+0.4 y_{2}$

subject to:

$$
\begin{array}{r}
y_{1}+2 y_{2} \geq 2 \\
1_{1}+y x_{2} \geq 3 \\
\text { 2) } y(i) \geq 0, i=1,2 .
\end{array}
$$

\section{Vertices}

Vertex $1=[1.6,0]$

Vertex $2=[0,1.6]$

Vertex $3=[0,0]$

\section{Epsilon}

$\varepsilon=0.01$

\section{Results}

$x(0)=[0.184,0.0937]$

$y(0)=[2,2]$

\section{Iteration 1}

Upper $Z(U Z)=2.4$, Lower $Z(L Z)=0.649$ 


$$
(U Z-L Z)=1.751, \text { is not }<\varepsilon
$$

New Constraint

3)

$$
2 x_{1}+3 x_{2} \geq 1.5245
$$

No feasible solution

\section{Iteration 2}

Upper $Z(U Z)=1.5245$, Lower $Z(L Z)=0: 649$

$$
(U Z-L Z)=0.8755, \text { is not }<\varepsilon
$$

New Constraint

3)

$$
2 x_{1}+3 x_{2} \geq 1.0868
$$

No feasible solution

\section{Iteration 3}

Upper $Z(U Z)=1.0868$, Lower $Z(L Z)=0.649$

$$
(U Z-L Z)=0.4377, \text { is not }<\varepsilon
$$

New Constraint

$$
\text { 3) } 2 x_{1}+3 x_{2} \geq 0.8679
$$

No feasible solution

\section{Iteration 4}

Upper $Z(U Z)=0.8679$, Lower $Z(L Z)=0.649$

$$
(U Z-L Z)=0.2189, \text { is not }<\varepsilon
$$

New Constraint

$$
2 x_{1}+3 x_{2} \geq 0.7584
$$

Feasible Solution: $x(1)=[0.3315,0.0323]$

\section{Iteration 5}

Upper $Z(U Z)=0.8679$, Lower $Z(L Z)=0.7584$

$$
(U Z-L Z)=0.1094, \text { is not }<\varepsilon
$$

New Constraint

3)

$$
2 x_{1}+3 x_{2} \geq 0.8132
$$

No feasible solution

\section{Iteration 6}

Upper $Z(U Z)=0.8132$, Lower $Z(L Z)=0.7584$

$$
(U Z-L Z)=0.0547, \text { is not }<\varepsilon
$$

New Constraint

3)

$$
2 x_{1}+3 x_{2} \geq 0.7858
$$

Feasible Solution: $x(2)=[0.3741,0.0129]$

\section{Iteration 7}

Upper $Z(U Z)=0.8132$, Lower $Z(L Z)=0.7858$

$$
(U Z-L Z)=0.0274, \text { is not }<\varepsilon
$$

New Constraint

3)

$$
2 x_{1}+3 x_{2} \geq 0.7995
$$

Feasible solution: $x(3)=[0.399,0.0005]$

\section{Iteration 8}

Upper $Z(U Z)=0.8132$, Lower $Z(L Z)=0.7995$ 


$$
(U Z-L Z)=0.0137, \text { is not }<\varepsilon
$$

New Constraint

3)

$$
2 x_{1}+3 x_{2} \geq 0.8063
$$

No feasible solution

\section{Iteration 9}

Upper $Z(U Z)=0.8063$, Lower $Z(L Z)=0.7995$

$$
(U Z-L Z)=0.0068, \text { is not }<\varepsilon
$$

Optimal Solution Found.

$Z^{*}=0.8029$

$x^{*}=x(3)=[0.399,0.0005]$

Multiplying the optimal solution by 10 gives the optimal solution of the original problem as

$Z^{*}=8.029$

$x^{*}=x(3)=[3.99,0.005]$

\section{Concluding Remarks}

The solution obtained by FRCA compares favorably with that obtained by the popular simplex algorithm. The optimal solution by simplex method is $Z^{*}=8 ; x^{*}=(4,0)$. Furthermore, the FRCA performs faster when the size of the feasible region is reduced. In the above illustration the number of iterations reduces from 12 to 9 .

\section{References}

E. O. Effanga. (2009). Alternative Technique for solving linear programming problems, Ph. D Thesis. Department of Mathematics/Statistics and Computer Science, University of Calabar - Nigeria (unpublished).

G. B. Dantzig, L. R. Ford (Jr) and D. R. Fulkerson. (1956). A primal-dual algorithm, linear inequalities and related systems. Annal of Mathematics study 38, Princeton University press, Princeton, New York.

G. Eiselt, C. Pederzolli and L. Sandblom. (1987). Operations Research. W de G. New York.

I. J. Lustig, R. E. Marsten and D. F. Shanno. (1994). Interior point methods for linear programming. Computational state of art. ORSA Journal on Computing, 6, 1 - 14.

J. Gondzio. (1995). Multiple centrality corrections in a primal - dual method for linear programming. TR No. 20 Logilab, HEC, Geneva, Section of Management Studies, University of Geneva, Bd. Carl - Vogt 102, 1211 Geneva, Switzerland.

L. A. Levin and B. Yammitsky. (1982). An old linear programming algorithm runs in polynomial time. Proceeding 23rd Annual Symposium of foundations of Computer Science. IEEF Computer Society, Long Beach. California, 327 - 328.

M. J. Todd and Y. Ye. (1990). A centered projective algorithm for linear programming. Mathematics of Operations Research, 15, 508 - 529.

N. Karmarkar. (1984). A New Polynomial Time Algorithm for linear programming. Combinatorial, 4, 373 - 395.

R. E. Marsten, R. Subramanian, M. Saltzman, I. J. Lustig and D. Shanno. (1990). Interior point methods for linear programming. Interfaces, 20, 105 - 116. 